\title{
High Angular Resolution Electron Backscatter Diffraction Studies of Tetragonality in Fe-C Martensitic Steels
}

\author{
Tomohito Tanaka ${ }^{1,2}$, Angus J Wilkinson ${ }^{1}$ \\ 1. Department of Materials, University of Oxford, Oxford, UK \\ 2. Advanced Technology Research Laboratories Nippon Steel \& Sumitomo Metal Corporation, \\ Shintomi, Futtsu, Japan
}

High (Angular) Resolution EBSD (HR-EBSD) was developed to measure the spatial variation in elastic lattice strain at the microstructural scale with strain sensitivity of $\sim 10^{-4}$ [1]. However, the measurements are relative to the strain state at the point from which the reference pattern is obtained. In some cases, this baseline reference strain state is well-known but in the general case it is not; the so-called reference pattern problem. One possible route to solving this problem and thus achieving absolute strain measurement is to use a simulated EBSD pattern as the reference pattern [2]. Here we explore recent steps towards this goal in technique development and through an important materials science challenge of mapping local variations in tetragonality within martensitic steels. Lath and plate martensite structures formed in low- to medium-carbon steels are of industrial significance. Martensitic phase transformation from the high temperature face centre cubic austenite to the body centre tetragonal martensite leads to complex hierarchical microstructures which balances lattice strain and interfacial contributions to energy. As the carbon content is increased X-ray diffraction studies have shown that the average c/a ratio of the martensite increases, while microscopy shows a refinement in microstructural lengthscale. There is, however, a lack of knowledge concerning the microscopic variation in tetragonality. We address this knowledge gap using some careful measurements using high resolution electron backscatter diffraction (HR-EBSD).

Several Fe-C martensitic steels were austenitised and water-quenched followed by liquid nitrogen cooling and then metallographically prepared for EBSD analysis. A small $\left(10 \times 10 \times 1 \mu \mathrm{m}^{3}\right)$ well-annealed interstitial free (IF) steel sample was mounted on each martensite block. Dynamical diffraction simulations [3] were conducted using the commercial ESPRIT DynamicS software (Bruker) for a BCC Fe crystal with intensities mapped onto a master simulation on a stereographic projection (fig. 1a). From this master simulation EBSD patterns for any crystal orientation and detector geometry were produced rapidly by interpolation to the required gnomonic projection (see fig. 1b). A differential evolution algorithm was used to find the detector geometry ( 3 degrees of freedom) and crystal orientation ( 3 degrees of freedom) that optimized the match between the experimental and simulated pattern intensities.

Having thus established and fixed the detector geometry a similar pattern matching was conducted between EBSD patterns from the martensitic steels and simulations conducted for BCT crystals at different c/a ratios. The start points for these optimizations made use of Hough-based orientation measurements assuming a BCC crystal and the possibility of the c-axis being along each of the three cube axes was checked in turn (fig. 2). Figure 3 shows how the absolute c/a ratio measured at a few local points in the martensitic steels increases with carbon content, as does the scatter in c/a ratio.

We then employed the HR-EBSD approach which uses cross-correlation at many sub-regions across the EBSD patterns is used to determine pattern shifts relative to some reference/seed patterns. Using the 
HR-EBSD mapping from the reference/seed points at which the detailed pattern matching measurements had been made allows more complete mapping of the tetragonality variation within the microstructure to be constructed (fig. 4).

\section{References:}

[1] AJ Wilkinson, G Meaden and DJ Dingley, Ultramicroscopy 106 (2006), p. 307.

[2] TB Britton et al, Ultramicroscopy 110 (2010), p. 1443-1453

[3] A Winkelmann in "Electron Backscatter Diffraction in Materials Science ( $2^{\text {nd }}$ Edition)" eds AJ Schwartz et al., (Springer US- New York) p. 21-33.


Figure 1. (a) master simulation for $\mathrm{Fe}$ at $30 \mathrm{keV}$ with intensities on stereographic projection, (b) interpolated intensities on gnomonic projection matched to (c) experimentally obtained EBSD from IF steel.

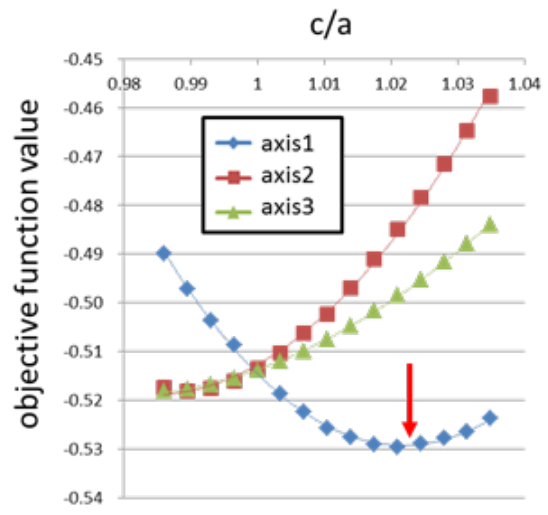

Figure 2. Pattern matching optimization identifying the $\mathrm{c}$-axis and c/a ratio. (Fe-0.77C).

Figure 3. c/a ratio measured at local points in martensites at for different carbon contents.

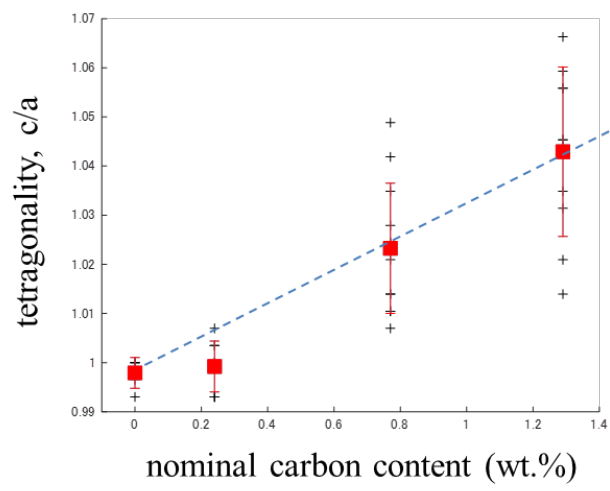

$\mathrm{c} / \mathrm{a}$

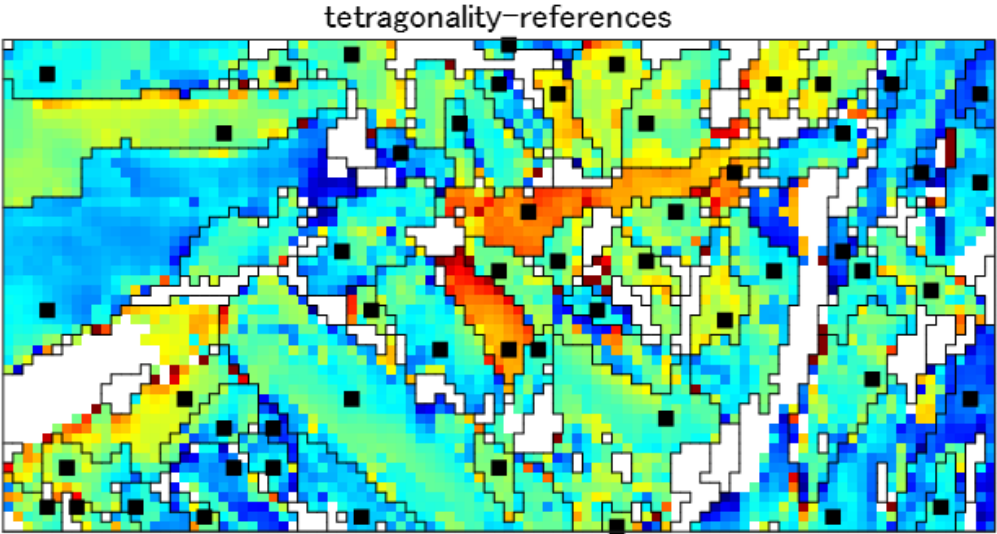

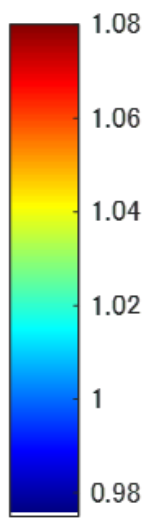

Figure 4. Map of c/a ratio constructed by combining pattern matching at seed points with HR-EBSD of variations across remainder of map. (Fe-0.77C) 